\title{
A RELEVÂNCIA DO CUIDADO ODONTOLÓGICO EM PACIENTES COM DIABETES: RELATO DE CASO ${ }^{1}$
}

\author{
THE RELEVANCE OF DENTAL CARE IN \\ PATIENTS WITH DIABETES: CASE REPORT \\ Danieli Londero da Silveira ${ }^{2}$, Tatiana Militz Perrone Pinto ${ }^{3}$, \\ Cátia Rubinstein Selistre ${ }^{4}$ e Juliana Romanini ${ }^{5}$
}

\section{RESUMO}

Indivíduos com diabetes mellitus tipo 2 (DM2) são mais suscetíveis a doenças da cavidade bucal, assim a atenção odontológica deve fazer parte da rotina de cuidados desses pacientes. Este trabalho tem como objetivo relatar um caso clínico, no qual um paciente do sexo masculino, 57 de anos de idade, com diagnóstico de dislipidemia, hipertensão arterial sistêmica e diabetes mellitus aos 42 anos de idade, após infarto agudo do miocárdio, compareceu ao Centro de Especialidades Odontológicas Santa Marta -Porto Alegre - RS, queixando-se que seus dentes ficavam amolecidos e depois caíam. Referiu que, em avaliações médicas e odontológicas anteriores, havia sido informado da relação do diabetes com a condição dentária e do risco de perda total dos dentes. Essa possibilidade fez com que o paciente se mobilizasse para a busca por tratamento especializado. Com diagnóstico de doença periodontal avançada, foi realizado tratamento de adequação bucal e tratamento periodontal. Recebeu orientação sobre as doenças bucais identificadas, importância do tratamento, da higiene bucal e dieta para a manutenção da saúde. O caso relatado evidencia a necessidade de acompanhamento odontológico para manutenção da saúde sistêmica desses indivíduos. O controle do distúrbio exige aquisição de conhecimento e atendimento adequado e resolutivo desses pacientes pelo cirurgião-dentista e equipe multiprofissional. Ao assumir um papel ativo no diagnóstico e no tratamento das condições bucais relacionadas ao diabetes, o dentista, associado às equipes multiprofissionais, pode contribuir para a manutenção da saúde e qualidade de vida de pacientes com esta doença.

Palavras-chave: Diabetes mellitus, Doença periodontal, Integralidade, Saúde bucal, Atenção Primária à Saúde.

\section{ABSTRACT}

Individuals with type 2 diabetes mellitus (DM2) are more susceptible to diseases of the oral cavity, so dental care should be part of the routine of care for these patients. This study aims to present a clinical case, in which a male patient, 57 years old, diagnosed with dyslipidemia, systemic arterial hypertension and diabetes mellitus at the age of 42, after acute myocardial infarction, attended the Center Dental Specialties Santa Marta - SMS Porto Alegre - RS, complaining that their teeth became soft and then fell out. He referred that, in previous medical and dental evaluations, he had been informed of the relationship between diabetes and the dental condition and the risk of total tooth loss. This possibility made the patient mobilize to seek specialized treatment.

\section{Relato de caso}

2 Autora. Odontopediatra e Mestre em Endocrinologia (UFRGS).E-mail:danielilondero29@gmail.com

3 Colaboradora. Docente do curso de Odontologia - Universidade Franciscana (UFN). E-mail: tatimilitz@hotmail.com 4 Colaboradora. Mestre em Odontologia - Universidade Federal do Rio Grande do Sul (UFRGS) e Cirurgiã-dentista no Centro de Especialidades Odontológicas - Santa Marta - Porto Alegre. E-mail: catia.selistre@gmail.com

5 Orientadora. Doutora em Estomatologia - Pontifícia Universidade Católica do Rio Grande do Sul (PUCRS) e Cirurgiã-dentista da Unidade de Odontologia Clínica e Hospitalar do HCPA. Estomalogia SMS Porto Alegre. E-mail: julianaromanini@hcpa.edu.br 
With diagnosis of advanced periodontal disease, oral adequacy treatment and periodontal treatment were performed. He received guidance on the identified oral diseases, the importance of treatment, oral hygiene and diet for maintaining health. The reported case highlights the need for dental follow-up to maintain the systemic health of these individuals. The control of the disorder requires the acquisition of knowledge and adequate and resolving care for these patients by the dentist and multidisciplinary team. By taking an active role in the diagnosis and treatment of oral conditions related to diabetes, the dentist, associated with multiprofessional teams, can contribute to maintaining the health and quality of life of patients with this disease.

Keywords: Diabetes mellitus, Periodontal disease, Integrality, Oral health, Primary Health Care.

\section{INTRODUÇÃO}

O diabetes mellitus (DM) configura-se hoje como uma epidemia mundial e um problema de saúde pública com grandes proporções quanto à magnitude e a transcendência, apesar dos progressos no campo da investigação e atenção aos pacientes (ASSUNÇÃO et al., 2001). Em 2019, aproximadamente 463 milhões de adultos entre 20 a 79 anos tinham diabetes e esse número pode aumentar para 700 milhões de pessoas até 2045 (IDF, 2019). O aumento na sua prevalência e incidência permite que o DM seja traduzido como um grande desafio aos serviços de saúde (MOORE et al., 2003). Assim, ações voltadas para a prevenção e controle da doença são fundamentais para a saúde em todo o mundo.

O DM é uma doença crônica que abrange um grupo de distúrbios metabólicos, que incluem redução na secreção de insulina, diminuição da utilização e aumento da produção de glicose. A insulina é o hormônio produzido pelo pâncreas, que age como uma chave para permitir que a glicose dos alimentos ingeridos passe da corrente sanguínea para as células do corpo para produzir energia (IDF, 2019). Não ser capaz de produzir insulina ou não a usar efetivamente leva ao aumento expressivo dos níveis de glicose no sangue caracterizado pela hiperglicemia. Está claramente demonstrado que, a longo prazo, altos níveis de glicose estão associados ao comprometimento de vários órgãos e tecidos (MAURI-OBRADORS et al., 2017).

Essas alterações fisiológicas têm potencial de diminuir a capacidade imunológica e a resposta inflamatória, aumentando a suscetibilidade às infecções. Além disso, a doença, quando não controlada adequadamente, pode apresentar complicações microvasculares (retinopatina, nefropatia, neuropatia) e macrovasculares (doenças cardiovasculares). Estes efeitos em locais densamente vascularizados e inervados também podem estar associados ao aumento de complicações orais (KAN et al., 2019; VERHULST et al., 2019; MAURI-OBRADORS et al., 2017).

Assim, sob a perspectiva de diferentes mecanismos, como hiperglicemia, resistência à insulina, dislipidemia, hipertensão arterial e disfunção imunológica, diferentes complicações orais fazem parte 
da rotina odontológica, incluindo doenças periodontais, cárie dentária, periodontite apical, doenças peri-implantares, má cicatrização de feridas, xerostomia, distúrbios do paladar, síndrome da boca ardente, lesões nas mucosas orais, câncer bucal e distúrbios temporomandibulares (VERHULST et al., 2019; MAURI-OBRADORS et al., 2017).

Os tecidos periodontais são as estruturas bucais mais afetadas pelo DM, sendo que a doença periodontal é considerada pela Organização Mundial da Saúde (OMS) como a sexta complicação crônica do distúrbio metabólico. A doença periodontal encontra-se presente em cerca de $75 \%$ dos casos e pode ser considerada como uma complicação microvascular do diabetes. Quanto mais cedo ocorre o aparecimento do diabetes e quanto maior for à duração da doença não controlada, o portador será mais suscetível a desenvolver a doença periodontal. Por isso, uma história aprofundada quanto ao aparecimento, duração e controle da doença é fundamental para o manejo clínico destes pacientes (KUO et al., 2008).

Vários mecanismos estão envolvidos na fisiopatogenia da doença periodontal associada ao DM. A suscetibilidade e progressão da infecção dos tecidos de proteção e sustentação dos dentes estão relacionadas com o descontrole metabólico, produtos de glicosilação avançados formados a partir da ligação da glicose a proteínas estruturais, deficiente resposta imune, redução da quimiotaxia de neutrófilos, função reduzida dos fibroblastos, alterações dos vasos sanguíneos, tecido conjuntivo, composição salivar e genéticas, como herança de determinados antígenos de histocompatibilidade (ALVES et al., 2007; KOCHER et al., 2018).

Uma vez que complicações orais crônicas podem ter efeitos negativos no controle glicêmico, os cirurgiões-dentistas devem estar aptos a diagnosticar, tratar e prevenir doenças da cavidade bucal em pacientes com diabetes. Ao assumir este papel, passam a ter uma visão geral das doenças sistêmicas associadas, estando atentos às principais manifestações orais e complicações relacionadas ao diabetes (KAN et al., 2019; KAUR et al., 2015).

No entanto, muitos pacientes desconhecem a associação entre diabetes e saúde bucal e não realizam acompanhamento odontológico. Avaliações bucais regulares, com ênfase na avaliação periodontal e na importância do controle do biofilme dental, associadas a mudanças no estilo de vida relacionadas ao controle da glicemia e outras práticas de autocuidado, podem efetivamente prevenir complicações orais em pacientes com diabetes. Assim, a atuação do cirurgião-dentista em equipes multiprofissionais, na prevenção e no gerenciamento das complicações orais é fundamental para essa população (KAN et al., 2019; KAUR et al., 2015; DE OLIVEIRA et al., 2016).

Pacientes com diabetes bem controlado podem ser tratados no consultório odontológico de maneira semelhante a pacientes sem diabetes. O atendimento odontológico aos pacientes com diabetes deve ser adaptado conforme as suas particularidades, levando-se em consideração o horário e o tempo dos procedimentos clínicos. Deve-se dar preferência por consultas no período da manhã, 
em que a insulina atinge seu nível máximo de secreção e os níveis endógenos de corticosteroides estão mais elevados, permitindo maior tolerância do paciente ao aumento da adrenalina e da glicemia, que resultam de situações de estresse relacionadas ao procedimento odontológico. Da mesma forma, consultas longas devem ser evitadas, pois podem levar o paciente a quadros de ansiedade. Recomenda-se que o paciente mantenha dieta habitual antes da consulta (AGGARWAL et al., 2018; DE OLIVEIRA et al., 2016).

Na presença de hipoglicemia, o cirurgião-dentista deve suspender o procedimento. Quando o diabetes estiver descompensado, aumenta o risco de complicações que podem limitar procedimentos odontológicos terapêuticos. Nessa situação, os tratamentos devem ser paliativos, buscando-se adiar procedimentos cirúrgicos até que o quadro metabólico do paciente estabilize. No entanto, focos de infecção dentária ativas precisam ser controlados, uma vez que podem ser a causa da descompensação metabólica, estabelecendo-se um ciclo vicioso (DE OLIVEIRA et al., 2016).

Acredita-se que a atuação dos cirurgiões-dentistas em equipes de saúde multidisciplinares, pode contribuir para a manutenção do bem-estar e qualidade de vida dos pacientes com esta doença e proporcionar uma elevação dos níveis de saúde da população (AERTS et al., 2004). Dessa forma, o objetivo deste trabalho é relatar um caso clínico de uma paciente diabetes mellitus que recebeu atendimento multidisciplinar e revisar a literatura sobre o tema.

\section{RELATO DE CASO CLÍNICO}

Paciente do sexo masculino, 57 anos, com diagnóstico de diabetes mellitus aos 42 anos de idade após infarto agudo do miocárdio. Permanece com dislipidemia e hipertensão arterial sistêmica. Tabagista ativo. Pais e irmão com histórico de diabetes, já falecidos. Encaminhado pela unidade de saúde para tratamento periodontal em função de risco de perda dentária por doença periodontal avançada. Na primeira consulta odontológica especializada, referiu que, em avaliações médicas e odontológicas anteriores, havia sido informado da relação do diabetes com a condição dentária e do risco de perda total dos dentes. Essa possibilidade fez com que o paciente se mobilizasse para a busca por tratamento especializado. Foi realizada anamnese e avaliação de exames laboratoriais trazidos pelo paciente. A fase ativa do tratamento odontológico durou nove meses, correspondendo ao intervalo das avaliações médicas 1 e 2 (Quadro 1). Há que se destacar algumas condições identificadas no exame clínico e radiográfico deste paciente (Figuras 1 a 3). 
Quadro 1 - Anamnese e informações clínicas do paciente.

\begin{tabular}{|c|c|c|}
\hline HISTÓRICO DE SAÚDE GERAL & Avaliação 1 & Avaliação 2 \\
\hline Diabetes mellitus & Ano 2019 & - \\
\hline Infarto agudo miocárdio & Ano 2019 & - \\
\hline Hipertensão Arterial Sistêmica & PA $130 \times 80 \mathrm{mmHg}$ & PA $130 \times 80 \mathrm{mmHg}$ \\
\hline HIV, Hepatites B e C & Sorologias negativas & - \\
\hline IMC & $29 \mathrm{~kg} / \mathrm{m}^{2}$ & $29 \mathrm{~kg} / \mathrm{m}^{2}$ \\
\hline \multicolumn{3}{|l|}{ MEDICAÇÕES EM USO } \\
\hline & Metformina $875 \mathrm{mg}$ & Metformina $875 \mathrm{mg}$ \\
\hline & Insulina NPH $20 \mathrm{UI}$ & Insulina NPH 24 UI \\
\hline & Regular 5-10 UI & Regular 5-10 UI \\
\hline & EPL 25mg & EPL 25mg \\
\hline & Omeprazol 20mg & Omeprazol 20mg \\
\hline & Isossorbida $40 \mathrm{mg}$ & Isossorbida $40 \mathrm{mg}$ \\
\hline & Metoprolol 200mg & Metoprolol 200mg \\
\hline & Enalapril 20mg & Enalapril 20mg \\
\hline & Anlodipina $10 \mathrm{mg}$ & Anlodipina $10 \mathrm{mg}$ \\
\hline & Hidralazina $25 \mathrm{mg}$ & Hidralazina $25 \mathrm{mg}$ \\
\hline & Diazepan & Diazepan \\
\hline & Fluoxetina $80 \mathrm{mg}$ & Fluoxetina 80mg \\
\hline & - & Sinvastatina $20 \mathrm{mg}$ \\
\hline \multicolumn{3}{|l|}{ EXAMES LABORATORIAIS } \\
\hline Glicose $(\mathrm{mg} / \mathrm{dL})$ & 257 & 204 \\
\hline Hemoglobina Glicada (\%) & 9,4 & 9,8 \\
\hline Colesterol total (mg/dL) & 227 & 261 \\
\hline Triglicerídeos (mg/dL) & 613 & 975 \\
\hline Creatinina $(\mathrm{mg} / \mathrm{dL})$ & 0,57 & 0,84 \\
\hline Ureia (mg/dL) & 30 & - \\
\hline HISTÓRICO DE SAÚDE BUCAL & $\begin{array}{c}\text { Avaliação 1 } \\
\text { Data: março/2019 }\end{array}$ & $\begin{array}{c}\text { Avaliação } 2 \\
\text { Data: dezembro/2019 }\end{array}$ \\
\hline Índice de placa visível (\%) & $40 \%$ & $32 \%$ \\
\hline Índice de sangramento gengival (\%) & $42 \%$ & $45 \%$ \\
\hline Profundidade de sondagem*(dp) & $5,5(2,6)$ & $5,4(2,4)$ \\
\hline Nível de inserção clínica*(dp) & $5,9(2,7)$ & $5,7(2,4)$ \\
\hline Sangramento à sondagem (\%) & $55 \%$ & $53 \%$ \\
\hline
\end{tabular}

*Valores médios pré e pós-intervenções periodontais 
Figura 1 - Radiografia panorâmica inicial realizada em março de 2019: perda óssea avançada (a); lesão de cárie cervical (b); patologia periapical (c). Sem outras patologias intraósseas.

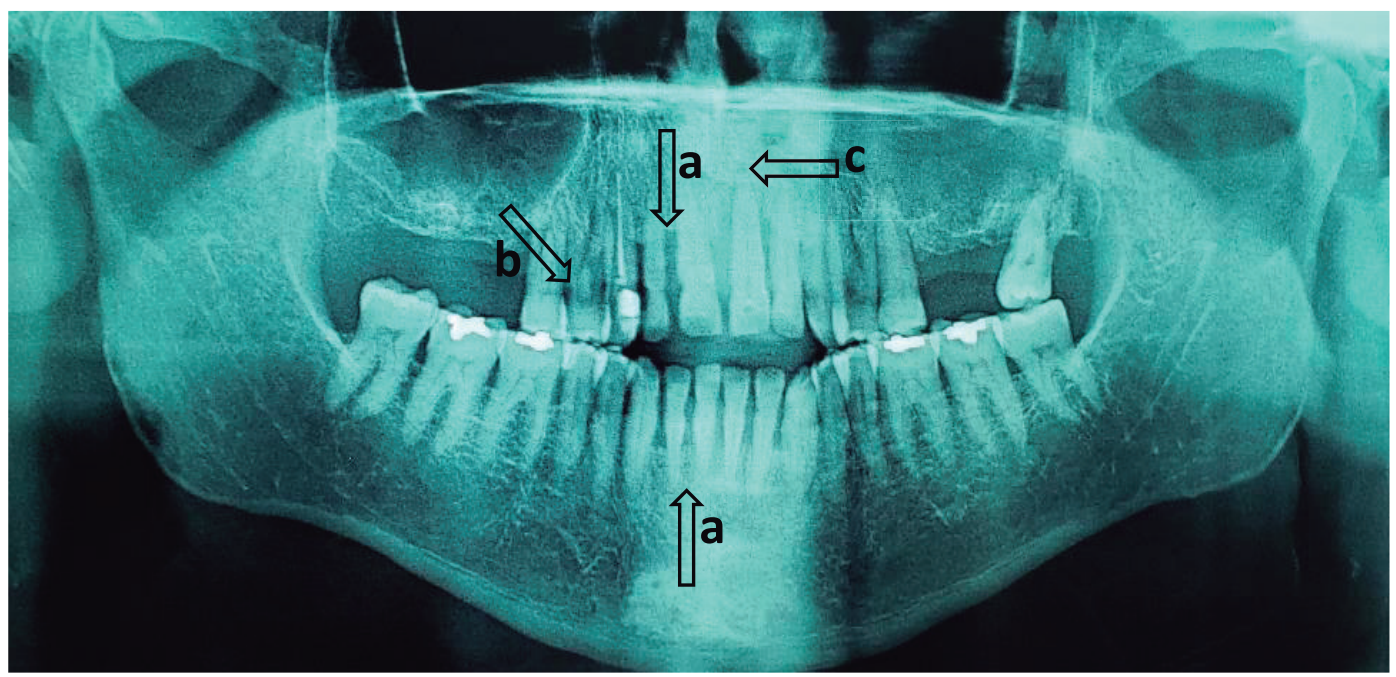

Figura 2 - Aspectos clínicos: perda de inserção, afastamento dentário, alteração de oclusão (d); saliva viscosa (e); biofilme dental (f).
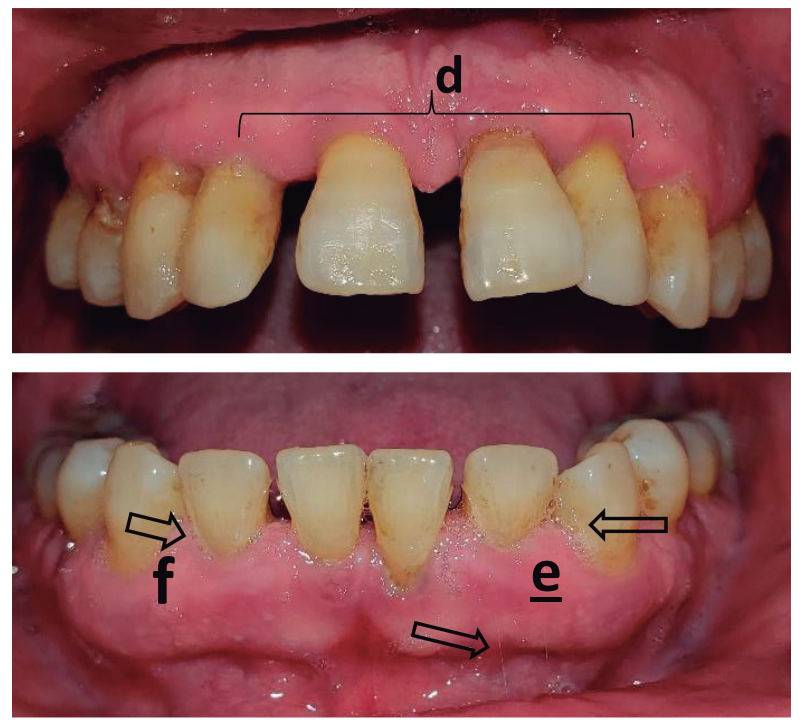
Figura 3 - Aspectos clínicos em março de 2019: sondagem periodontal (g) para avaliação da perda de inserção, recessão gengival (h) e sinais inflamatórios. O limitado controle do biofilme (f), associado à redução do fluxo salivar, favorece o desenvolvimento de lesões cariosas cervicais (i) que, por sua vez, aumenta a retenção de biofilme.

Ciclo que precisa ser interrompido pelas ações de higiene oral e dieta: autocuidado. Herpes bucal (j).

Aspecto radiográfico em dezembro de 2019: endodontia realizada (k).
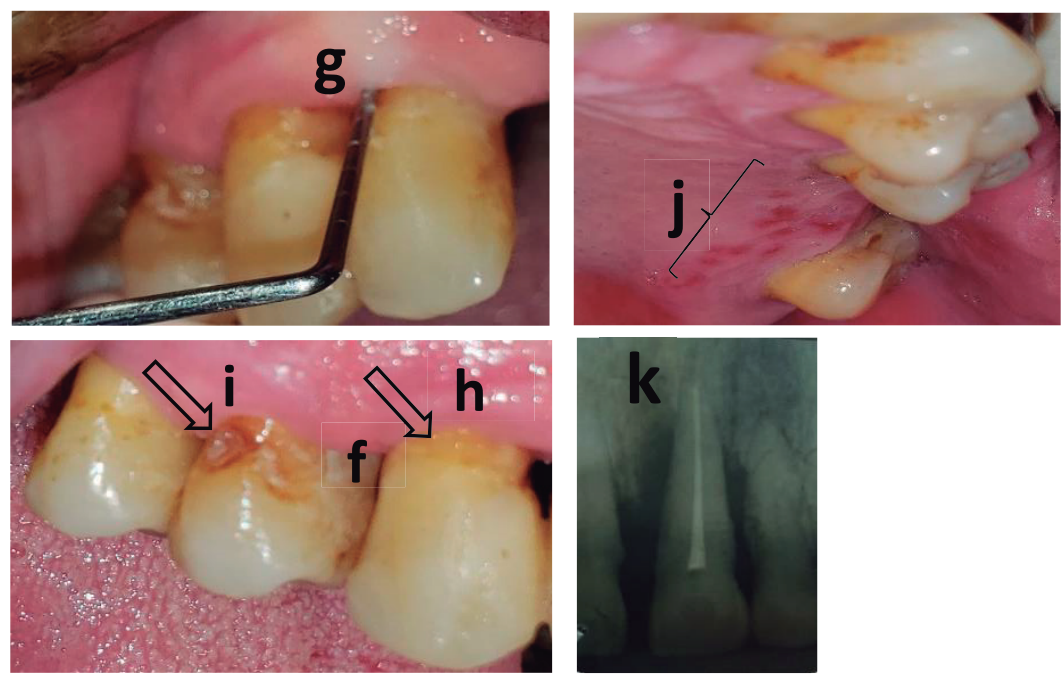

Após anamnese e avaliação clínica e radiográfica, o diagnóstico principal foi de periodontite generalizada associada à desordens sistêmicas que influenciam a patogênese das doenças periodontais. O paciente ainda apresentava lesões cariosas, redução do fluxo salivar e infecções oportunistas eventuais. Foi estabelecido plano de tratamento, iniciando pela remoção dos fatores retentivos de biofilme (cavidades e cálculo). Num segundo momento, realizadas as raspagens e alisamentos subgengivais, com abordagem convencional e acesso cirúrgico em alguns elementos. Concomitantemente, foi realizado o tratamento endodôntico do incisivo central. Sempre que indicado, para a realização dos procedimentos sob anestesia local, foi utilizada prilocaína 3\% com o vasoconstritor felipressina, na concentração 1:100.000.

Nos procedimentos cruentos, foi adotado o uso de antibioticoterapia com amoxicilina 500mg a cada 8 h por 7 dias. O paciente foi orientado a usar paracetamol 500mg a cada $4 \mathrm{~h}$, para dor leve. Para o controle químico do biofilme, foi utilizada solução de clorexidine $0,12 \%$, bochecho, 12/12h (sempre sem álcool, para reduzir danos à mucosa). Com foco no autocuidado, destacando a fisiopatologia das doenças, o paciente recebeu instrução de higiene oral com fio dental e escova macia, pelo menos duas vezes ao dia. Foi orientado também a hidratar-se e estimular a salivação (chá de camomila e sucos cítricos) e se necessário, poderia lançar mão de chiclete sem açúcar. A cada consulta o reforço de educação era realizado. 


\section{DISCUSSÃO}

Definida como doença metabólica de etiologia múltipla, o DM apresenta como característica principal a hiperglicemia crônica, que decorre da ausência, deficiência e/ou resistência à ação do hormônio insulina, sintetizado pelas células beta-pancreáticas (MILECH et al., 2016). Segundo o Caderno de Atenção Básica de Diabetes Mellitus, os portadores devem ser encaminhados para avaliação e cuidado odontológico, pois as infecções agudas e condições inflamatórias podem aumentar a taxa de glicose (BRASIL, 2013).

Há evidências de que pacientes com diabetes mal controlado, o controle deficiente do biofilme, associado à redução do fluxo salivar, pode aumentar também o risco à cárie e ambas as doenças podem levar à perda dentária, alterando padrões de mastigação e comprometendo a saúde geral. Além disso, o DM está associado com uma maior probabilidade de desenvolver certas desordens da mucosa oral, sendo comuns lesões orais como candidíase, glossite romboide mediana, queilite angular, herpes bucal e líquen plano. Ainda podem estar associadas a piora do controle glicêmico o uso de próteses, xerostomia e desregulação imunológica (COSTA et al., 2016).

No caso clínico apresentado, os aspectos da periodontite percebidos pelo paciente, como sangramento, sinais inflamatórios e mobilidade são comuns quando há pobre controle glicêmico, pois o diabetes é um fator de risco para a doença periodontal, e a doença periodontal, quando presente, leva ao estímulo da resposta inflamatória, aumentando a resistência dos tecidos à insulina, o que provoca a piora do controle glicêmico. O teste de hemoglobina glicada (HbAlc) reflete a concentração dos níveis de glicose sanguínea nos últimos 2-3 meses, portanto, trata-se de um exame importante tanto para o diagnóstico quanto para o seguimento do paciente com diabetes (SBD, 2019). Há de se destacar neste caso que a piora nos níveis de hemoglobina glicada (HbAlc), deve-se ao fato do paciente não aderir adequadamente à terapia proposta, refletindo consequentemente em pouca melhora na sua condição clínica periodontal. Nos pacientes com diagnóstico de periodontite, a doença deve ser tratada para contribuir na melhora do controle glicêmico (raspagem subgengival convencional ou por acesso cirúrgico) (MAURI-OBRADORS et al., 2017).

Também é comum a modificação da flora bucal, havendo uma predominância de Candida albicans, colônias de estreptococos hemolíticos e estafilococos, com tendência a candidíase oral e queilite angular em indivíduos com diagnóstico de DM (FANG et al., 2006). Para o tratamento da candidíase, podem ser usados nistatina $100.000 \mathrm{UI} / \mathrm{ml}$ (suspensão oral) ou miconazol 2\% (gel oral), para as situações que podem ser aplicadas o uso tópico três a quatro vezes ao dia. Eventualmente, há necessidade de uso sistêmico de fluconazol $150 \mathrm{mg}$ (um comprimido ao dia, por 10 dias). Sempre que realizado uso sistêmico, ajustar dose em pacientes com insuficiência renal. Assim como no caso mencionado, em quadros de dor leve a moderada no pós-operatório, pode-se prescrever a dipirona e o paracetamol 
nas mesmas dosagens e posologias utilizadas em pacientes sem diabetes. Anti-inflamatórios esteroidais devem ser evitados. O diclofenaco é uma opção de uso, se indicado (COSTA et al., 2016).

O paciente do caso em questão, precisou realizar procedimentos invasivos e cruentos. Sendo assim foram adotadas medidas e cuidados necessários para evitar infecções e complicações. A literatura evidencia que em pacientes com diabetes descompensado, o uso de adrenalina tem sido contraindicado durante procedimentos cirúrgicos, pois este hormônio provoca a quebra de glicogênio em glicose podendo resultar em hiperglicemia. Dessa forma para estes pacientes, é mais sensato utilizar o anestésico prilocaína com o vasoconstritor felipressina, pelo fato de ser um vasoconstritor hormonal de pequeno impacto sobre o sistema cardiovascular. No entanto, em pacientes com diabetes compensado, o uso de anestésico com vasoconstritor adrenérgico é permitido (mepivacaína 2\% com adrenalina 1:100.000 ou lidocaína 2\% com epinefrina 1:100.000 - volume máximo de 5,4 $\mathrm{ml}=3$ tubetes) (COSTA et al., 2016).

Atenção também deve ser dada a prescrição de profilaxia antibiótica antes do procedimento cirúrgico, pois a hiperglicemia tende a produzir um aumento no sangramento pós-operatório, devido uma dissolução excessiva do coágulo (COSTA et al., 2016). Neste caso, a utilização de antibióticos foi baseada na ausência de controle metabólico do paciente. Apesar de não existir um consenso na literatura em relação ao uso de terapias antimicrobianas sistêmicas para o tratamento de periodontite em paciente com diabetes, essa revisão sistemática recente mostrou benefícios clínicos em relação a redução de profundidade de sondagem, sendo considerada uma terapia adjuvante benéfica ao tratamento mecânico e teve indicação justificada pela ausência de controle glicêmico dos pacientes com diabetes (SOUTO et al., 2018). Ressalta-se que o uso de antibióticos como profilaxia, dependerá da condição clínica do paciente e do procedimento a ser realizado, além de atendimentos de urgência nos quais não seja possível determinar a classificação exata de risco apresentado pelo paciente. Nos procedimentos de rotina, sem risco de infecção, não há indicação do uso de antibióticos. Como parte da terapêutica, o antibiótico é indicado para os pacientes com diabetes compensado quando houver infecção presente. Quando mais suscetíveis, em função de diabetes mal ou não controlado, sempre há indicação de uso de antibióticos nos procedimentos cirúrgicos ou nas situações com risco de infecção e retardo de cicatrização (AGGARWAL et al., 2018; COSTA et al., 2016).

O caso relatado e estudos levantados trazem à luz a discussão de que alterações bucais são frequentes em pacientes com diabetes, sugerindo a necessidade de cuidados com a saúde bucal. Apesar da relevância deste caso clínico, devemos enfatizar que este trabalho apresenta limitações inerentes aos estudos de caso, como a impossibilidade de generalizar os resultados obtidos, pois pode não representar a população geral. Contudo, é importante estabelecer o controle de glicemia e esses pacientes precisam conhecer as doenças que podem ocorrer na cavidade bucal em função da doença sistêmica. A manutenção periódica odontológica profissional é recomendada: controle supragengival com controle do biofilme dental bem como receber orientação quanto aos cuidados de higiene bucal para manutenção da saúde, com foco no autocuidado (KAN et al., 2019). 


\section{CONSIDERAÇÕES FINAIS}

Dados epidemiológicos demonstram que o diabetes constitui um importante problema de saúde pública. A frequente procura de atendimento na Atenção Primária à Saúde (APS) e a importância do controle metabólico para evitar complicações orais e comorbidades associadas, exigem aquisição de conhecimento atualizado em relação à doença, suas consequências e necessidades para prestação de atendimento adequado a essa população.

Sendo assim, a garantia da integralidade do cuidado, por equipe multiprofissional, busca minimizar os riscos de complicações orais e evitar prejuízo ao controle do diabetes. Ao assumir um papel ativo no diagnóstico e no tratamento das condições bucais relacionadas ao diabetes, o dentista, associado às equipes multiprofissionais, pode contribuir para a manutenção do bem-estar e qualidade de vida dos pacientes com esta doença.

\section{CASO CLÍNICO}

As imagens e as informações foram usadas com o consentimento do paciente.

\section{REFERÊNCIAS}

ASSUNÇÃO, M. C. F.; SANTOS, I. S.; GIGANTEA, D. P. Atenção Primária em Diabetes no Sul do Brasil: Estrutura, Processo e Resultado. Revista de Saúde Pública, v. 35, n. 1, p. 88-95, 2001.

International Diabetes Federation (IDF). IDF Diabetes Atlas. 9th ed. 2019. Disponível: https:// diabetesatlas.org/en/resources/

MOORE, P.; ZGIBOR, J. C.; DASANAYAKE, A. P. Diabetes: A Growing Epidemic of All Ages. JADA, v. 134, p. 11-15, 2003.

KAN, S.; FISCHER, R. G.; DIAS, A. T. et al. Periodontia e Implantodontia Contemporânea. São Paulo: Quinta Editora, p. 690, 2019.

VERHULST, M. J. L.; LOOS, B. G.; GERDES, Teeuw W. J. Evaluating all potential oral complications of diabetes mellitus. Frontiers in Endocrinology, v. 18, p. 10-56, 2019.

MAURI-OBRADORS, E. et al. Oral manifestations of diabetes mellitus. A systematic review. Medicina Oral Patologia Oral y Cirugia Bucal, v. 22, n. 5, p. 586-594, 2017. 
KUO, L. C.; POISON, A. M.; KANG, T. Associations between periodontal diseases and systemic diseases: A review of the inter-relationships and interactions with diabetes, respiratory diseases, cardiovascular diseases and osteoporosis. Public Health, London, v. 122, n. 4, p. 417-433, 2008.

ALVES, C.; ANDION, J.; BRANDÃO, M.; MENEZES, R. Mecanismos Patogênicos da Doença Periodontal Associada ao Diabetes Melito. Arquivos Brasileiros de Endocrinologia Metabólica, v. 51, n. 7, p. 1050-1057, 2007.

KAUR, S.; KAUR, K.; RAI, S.; KHAJURIA, R. Oral health management considerations in patients with diabetes mellitus. Archives of Medicine and Health Sciences, v. 3, n. 1, p. 72-79, 2015.

KOCHER, T. et al. Periodontal complications of hyperglycemia/diabetes mellitus: Epidemiologic complexity and clinical challenge. Periodontology 2000, v. 78, p. 59-97, 2018.

AGGARWAL, A. et al. Dental management of diabetes mellitus: Review of literature. Journal of Advanced Oral Research, v. 7, n. 3, p. 73-77, 2018.

COSTA, R. N. et al. O Paciente Diabético na Clínica Odontológica: Diretrizes Para o Acolhimento e Atendimento. Revista Brasileira de Ciências da Saúde, v. 20, n. 4, p. 333-340, 2016.

SOCIEDADE BRASILEIRA DE DIABETES (SBD). Diretrizes da Sociedade Brasileira de Diabetes (2019-2020). São Paulo: AC Farmacêutica, 2019.

SOUTO, M. L. S. et al. Efficacy of systemic antibiotics in nonsurgical periodontal therapy for diabetic subjects: a systematic review and meta-analysis. International Dental Journal, v. 68, p. 207-220, 2018.

FANG, L.; FAZIO, R. C.; SONIS, S. T. Princípios e Prática de Medicina Oral. Guanabara Koogan, 2 ed, 508p, 2006.

DE OLIVEIRA, T. F. et al. Conduta odontológica em pacientes diabéticos: considerações clínicas. Odontologia Clínico-Científica, v. 15, n. 1, p. 1-5, 2016.

AERTS, D.; ABEGG, C.; CESA, K. O papel do cirurgião-dentista no Sistema Único de Saúde. Ciência \& Saúde Coletiva, v. 9, n. 1, p. 131-138, 2004. 
MILECH, A. et. al. Diretrizes da Sociedade Brasileira de Diabetes (2015-2016). Organização José Egidio Paulo de Oliveira, Sérgio Vencio - São Paulo: A.C. Farmacêutica, 2016.

BRASIL, Ministério da Saúde. Saúde Bucal - Caderno de Atenção Básica no 36. Brasília-DF, 2013. Disponível em: https://bit.ly/3bhRbxc. Acesso em: 20 nov. 2020. 